\title{
Relato de Experiência sobre a Criação de um Clube de Desenvolvimento de Jogos com Foco em Habilidades de Pensamento Computacional
}

\author{
Raphael A. de Lima, Eduardo F. de Sá, André P. Porto, \\ Rodrigo L. Rodrigues, José A. da Silva. \\ Departamento de Computação - Universidade Federal Rural de Pernambuco \\ Recife - PE - Brasil \\ raphaelalbino_@hotmail.com, eduardo.fsa2@ufrpe.br, \\ andre.porto@ufrpe.br, rodrigomuribec@gmail.com, \\ zealexandrejulio@gmail.com
}

\begin{abstract}
This article reports the experience of a group of a Computing course undergraduates in a public technical high school. After evaluating the context that the school resides, several activities were stimulated in order to improve student's school performance in computer programming and solving problems. Their affinities and previous knowledge about digital games were used to apply an inverted classroom methodology in order to teach the concepts of computational thinking. The blending of a theme that motivates students with programming concepts has shown positive results in order to increase student's familiarity with game development and consequently their resourcefulness in programming logic.
\end{abstract}

Resumo. Este trabalho relata a experiência de um grupo de graduandos de um curso de Licenciatura em Computação em uma escola pública de ensino médio técnico. Foram estimuladas diversas atividades com o intuito de melhorar o desempenho escolar dos alunos nas competências de programação. Suas afinidades e conhecimentos prévios sobre o tema de jogos digitais foram utilizados para aplicar uma metodologia de sala de aula invertida com o intuito de ensinar os conceitos de pensamento computacional. A mistura de um tema que instiga os alunos com conceitos de programação mostrou resultados positivos no sentido de aumentar a familiarização dos alunos com o desenvolvimento de jogos, e consequentemente, a lógica de programação.

\section{Introdução}

Ao ingressar em cursos de computação, geralmente os estudantes deparam-se com disciplinas relacionadas a lógica de programação. Boa parte destes alunos apresentam dificuldades com os conceitos iniciais dada a pouca familiaridade com o tema durante $o$ período escolar de nível fundamental e médio, o que leva ao aumento do número de retenção em cursos desta natureza (SILVEIRA et al., 2018).

De acordo com Jerkins (2002, apud SOUZA, 2013), as principais causas de baixo rendimento nas disciplinas de programação são: $O$ baixo nível de abstração, a 
falta de habilidade de resolução de problemas, metodologias de dinâmica que não estimula o aprendizado, entre outros. O foco deste projeto é aplicar a metodologia de sala de aula invertida para buscar indícios de como o aprendizado do aluno pode melhorar significativamente com um acompanhamento de monitores que visam dar o máximo de atenção, tirar dúvidas em sala de aula não sanadas devido às limitações de tempo e conteúdos dados pelo currículo do curso. Um dos pontos a serem explorados pela dinâmica de clube é o ambiente lúdico e descontraído que, em combinação com a sala de aula invertida, potencialize a motivação dos alunos. Ao utilizar esta metodologia buscamos a otimização do tempo em sala de aula com o uso de atividades reflexivas e de construção do conhecimento através da resolução de atividades propostas com o conteúdo adquirido previamente.

Foi escolhido o desenvolvimento de jogos como alvo de estudo por sua temática ser de grande interesse por parte dos jovens e por sua ligação ao cotidiano dos estudantes. É nítida a crescente utilização de soluções gamificadas, não só se tratando de jogos de entretenimento assim como de simulações da vida real. Ao criar uma correlação entre as diferentes mecânicas de jogos vivenciados pelos alunos com a maneira como eles são codificados, foi possível aplicar o pensamento computacional à prática de programação, buscando um contexto aplicável e prazeroso de ser explorado.

\section{Trabalhos relacionados}

Percebe-se na literatura que o uso de jogos para o ensino de programação não é uma novidade e continua demonstrando impactos positivos na aprendizagem.

\subsection{Oficinas de Jogos Digitais}

O principal objetivo do trabalho de Marques et al. (2011, p. 1139) foi de investigar e propor técnicas para a introdução de noções de programação para alunos do ensino médio, sendo uma delas o uso da programação de jogos. Ainda de acordo com os autores, iniciar a programação pelos jogos serve também como fator motivacional que contribui para aumentar o interesse dos alunos do ensino médio na área de computação. Aqueles que ainda não tinham um contato direto com o computador puderam ser motivados pela oficina.

De forma análoga, Andrade et al. (2016, p. 1135) descrevem: “A partir dos resultados alcançados, levantamos mais indícios para reforçar o argumento que $\mathrm{o}$ desenvolvimento de jogos digitais pode ter um impacto positivo quando adotado como fator motivacional no processo de ensino-aprendizagem.". Também nota-se que a metodologia de gamificação foi utilizada de forma competitiva: "Nesta edição, a competitividade entre equipes foi mais estimulada. Ocorreu a minimaratona de programação e o desafio de publicidade dos jogos desenvolvidos nessa minimaratona." (ANDRADE et al. 2016, p. 1131). Esta última proposta foi bastante similar ao trabalho aqui relatado, tendo em vista que uma futura etapa no clube de desenvolvimento envolveria a publicação dos jogos projetados pelos alunos.

Nos trabalhos em que já foram usados, os jogos serviram tanto como atrativo quanto meio de aprendizado rico e importante para o crescimento dos alunos. Nota-se também na literatura a satisfação por parte dos alunos, ou seja, além de demonstrarem resultados práticos, estão contentes com o trabalho realizado. Sendo assim, é notável a versatilidade do uso de jogos na educação, podendo ser usados tanto no ensino médio quanto no ensino superior. 


\subsection{Sala de Aula Invertida (Flipped Classroom)}

A prática deste conceito valorizou a diminuição do tempo de aulas expositivas de tal forma que os monitores do clube tiveram mais tempo para auxiliar o desenvolvimento das tarefas baseado no conteúdo disponibilizado previamente na internet. Segundo (Carvalho, 2014),

\footnotetext{
"O flipped classroom é um modelo de ensino que está ganhar força na comunidade educativa, ainda que não seja um modelo de ensino aprendizagem novo. A diferença está no fato de que as tecnologias da web e dos meios digitais estarem a aumentar o potencial deste modelo de forma exponencial. É uma abordagem ao processo de ensino-aprendizagem na qual se emprega a tecnologia para inverter o papel tradicional do tempo de aula, aqui os alunos são expostos a conceitos fora da sala de aula, geralmente através da observação e análise de vídeos. O tempo de sala de aula é então utilizado para fazer o difícil trabalho de assimilar esses novos saberes, através de estratégias como a resolução de problemas, discussão ou debates, sendo integralmente dedicado a experiências de aprendizagem ativas. Assim, recorrendo ao uso de tecnologias na partilha de dúvidas e curiosidades, a organização da sala de aula altera-se, passando os aprendentes para a posição central, previamente ocupada pelo professor, promovendo uma maior dinâmica em sala de aula. Os alunos não aprendem a partir das tecnologias, mas as tecnologias podem apoiar a construção de significados por parte dos alunos".
}

\section{Metodologia}

\subsection{Seleção dos Alunos e Materiais Utilizados}

Os alunos dos $1^{\circ}$ e $2^{\circ}$ s anos do ensino médio de tempo integral do curso técnico de Desenvolvimento de Sistemas da ETE do Porto Digital, interessados em participar do projeto, passaram por um processo de seleção que procurou captar principalmente o interesse prévio pelo assunto. Dentre os principais questionamentos procurou-se perceber: a afinidade com diferentes tipos de jogos; a curiosidade dos mesmos sobre ferramentas utilizadas na produção e desenvolvimento de jogos; da possibilidade de praticar os assuntos em casa. Também foi considerada indicação de professores das disciplinas do currículo técnico, no sentido de apontar os alunos mais interessados por desenvolver e por fim, foram escolhidos 15 alunos por turma.

Como material principal, foram utilizadas máquinas do tipo netbook - chamadas de tablets na ocasião e comumente utilizados na rede pública do Estado - que desfrutavam de tela sensível ao toque porém de baixa qualidade, baixo poder de processamento, apenas 10 polegadas, mas de boa capacidade de bateria a ponto de não precisarem de carregadores ligados à tomada durante as atividades, dando liberdade aos participantes de se movimentarem livremente no espaço da biblioteca que foi o local em que a escola alocou o clube. Já como ferramenta de desenvolvimento, optou-se pelo Unity $3 \mathrm{D}^{\mathrm{TM}}$, dado que é uma ferramenta já consagrada no cenário de jogos e ter uma comunidade numerosa e ativa que facilita a pesquisa por materiais e soluções na internet.

\subsection{Recursos Didáticos}

A partir das condições acima, foram produzidas até então 14 videoaulas, organizadas de tal maneira que os assuntos teóricos de programação e pensamento computacional fossem prioritariamente intercalados com as aulas práticas relacionadas a ferramenta, sempre objetivando uma aplicação de conceitos de forma convergente. Foram pensados inicialmente os seguintes temas conforme o quadro a seguir. 


\section{Quadro 1. Temas iniciais}

\begin{tabular}{|c|c|c|}
\hline Encontro & $\begin{array}{l}\text { Programação e Pensamento } \\
\text { Computacional }\end{array}$ & Aplicação da Ferramenta \\
\hline 1 & $\begin{array}{c}\text { Noções de Algoritmo e Pensamento } \\
\text { Computacional }\end{array}$ & Introdução ao Unity3D TM \\
\hline 2 & Scripts e Tipos de Variável & \\
\hline 3 & $\begin{array}{c}\text { Operadores Relacionais, Lógicos e } \\
\text { Aritméticos. }\end{array}$ & Aplicando Colisões \\
\hline 4 & Estruturas Condicionais & $\begin{array}{c}\text { Efeito Parallax e Movimentação } \\
\text { do Player }\end{array}$ \\
\hline 5 & Instantiate & Prefabs \\
\hline 6 & Funções e Procedimentos & Inputs \\
\hline 7 & \multicolumn{2}{|c|}{ Contador de Tempo } \\
\hline 8 & \multicolumn{2}{|c|}{ Triggers e Collision++ } \\
\hline 9 & Selecionando Inimig & s com Vetores \\
\hline
\end{tabular}

O encontro presencial foi idealizado para que não tivesse um cunho tutorial, partindo para esta abordagem apenas quando fosse realmente necessário e somente nas temáticas da ferramenta utilizada. Como objeto de estudo, a primeira mecânica de jogo a ser abordada foi a endless runnerı, sendo na nossa visão a que mais se adequou aos tipos de conteúdo abordados. As videoaulas foram disponibilizadas numa lista de reprodução do YouTube ${ }^{\mathrm{TM}_{2}}$ no modo não-listado, liberando um ou dois vídeos a cada semana dependendo da demanda da aula. A visualização destas foi obrigatória para que os alunos participassem das aulas práticas com a duração de $1 \mathrm{~h} 30$ em que todos os conhecimentos nelas requisitadas fossem adquiridas previamente através dos vídeos. Também foi recomendado que os alunos pesquisassem sobre os assuntos tratados e que tirassem suas dúvidas com os bolsistas, seguindo a metodologia de sala de aula invertida.

\section{Execução do Projeto e Relatos}

Como dito anteriormente, o local disponibilizado pela escola para a prática do clube. Já com relação aos horários, foram usadas as aulas de E.D. (Estudo Dirigido) nas quais o aluno tem o tempo de duas aulas de 50 minutos para realizar a atividade que julgar relevante. Sendo assim, separaram-se duas turmas, uma com os alunos do $1^{\circ}$ ano, às sextas-feiras e outra com os do $2^{\circ}$ ano, às terças-feiras.

No que tange o quantitativo de alunos participantes, após o primeiro encontro com a finalidade de apresentar o projeto de fato, continuaram entre 10 e 12 alunos por turma. Esta medida foi diminuindo gradativamente. Até a finalização deste relato, o clube encontra-se com 6 alunos por turma e totalizados 10 encontros por turma.

\subsection{Videoaulas}

Percebeu-se que a quantidade inicial de visualizações não condizia com a quantidade de

1 Mecânica de jogo que consiste em um único estágio que termina apenas quando o personagem morre.

2 Disp. em <https://www.youtube.com/channel/UCdkTfJK3Fw_KqX7f_fFSWMA/playlists> 
alunos, mesmo que tenha sido dada ênfase no primeiro encontro sobre a importância dos vídeos e de que a proposta de aulas tutoriais na biblioteca não eram bem-vindas. Portanto foram criadas duas abordagens para resolver o problema.

Inicialmente foi dado como obrigatório o retorno da atividade via ambiente virtual de aprendizagem, o Google Classroom $^{\mathrm{TM}}$, mesmo ambiente onde foi disponibilizado o link das aulas, algumas recomendações pré-aula e mensagens oficiais do clube, além de ser uma maneira de gerenciarmos as turmas. Também foi utilizado o WhatsApp ${ }^{\mathrm{TM}}$ para comunicações informais, disponibilizado dentro do próprio Classroom. Mesmo assim, a justificativa da desistência de um dos alunos foi a mesma que a maioria dos alunos usou para não assistir a alguns vídeos, o excesso de atividades na escola que é de tempo integral.

\subsection{Atividades na Biblioteca e Recursos Didáticos}

As atividades na Biblioteca foram produtivas, porém não tanto quanto se esperava. Devido à baixa quantidade de visualizações dos vídeos dados na semana anterior para que fossem estudados até o dia da atividade, acabou tornando os encontros em aulas expositivas. Para resolver o problema, escolhemos utilizar o Kahoot! ${ }^{\mathrm{TM}_{3}}$ para incentivar a visualização dos vídeos e criar uma competição. Foi utilizado um projetor que acabou também melhorando a interação monitor/aluno por conseguir atingir mais alunos de uma só vez na resolução dos questionamentos, além de servir como recurso nos momentos em que os tutoriais fossem necessários. A aceitação do método foi imediata e bastante motivador para os alunos, aumentando a quantidade de visualizações.

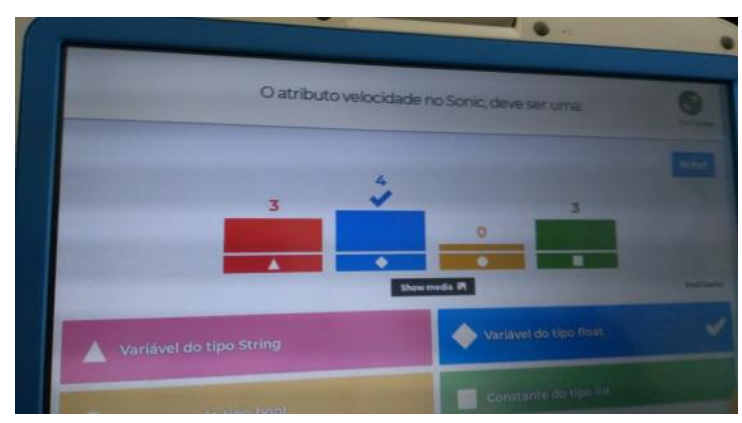

Figura 1. Exemplo de atividade com Kahoot! ${ }^{\mathrm{TM}}$ aplicando a vivência com jogos no contexto da programação.

Outro desafio presente no decorrer do clube foi a inadequação do uso ferramenta Unity3 $\mathrm{D}^{\mathrm{TM}}$ para rodar nos tablets. A ferramenta mostrou-se instável, resultando na ocorrência de vários bugs4 e fechamentos abruptos, sendo possivelmente um fator agravante de desistência. Foram feitas diversas intervenções na tentativa de otimizar o desempenho, com relativo sucesso. Também foi cogitada uma mudança de plataforma para algum programa que exigisse menos processamento das máquinas, porém tal mudança não ocorreu devido o fato de os alunos já estarem familiarizados com o Unity3 $\mathrm{D}^{\mathrm{TM}}$ além de que outra ferramenta significaria migrar para outra linguagem de programação, o que atrasaria o andamento das aulas.

3 É um jogo conectado de questionários eletrônico que gratifica com pontos e classifica os que responderem corretamente de forma mais rápida. Disponível em <https://www.kahoot.com/>.

4 Erros na execução do sistema, tais como o travar ou apresentar desempenho mínimo tornando o programa inutilizável por alguns segundos. 

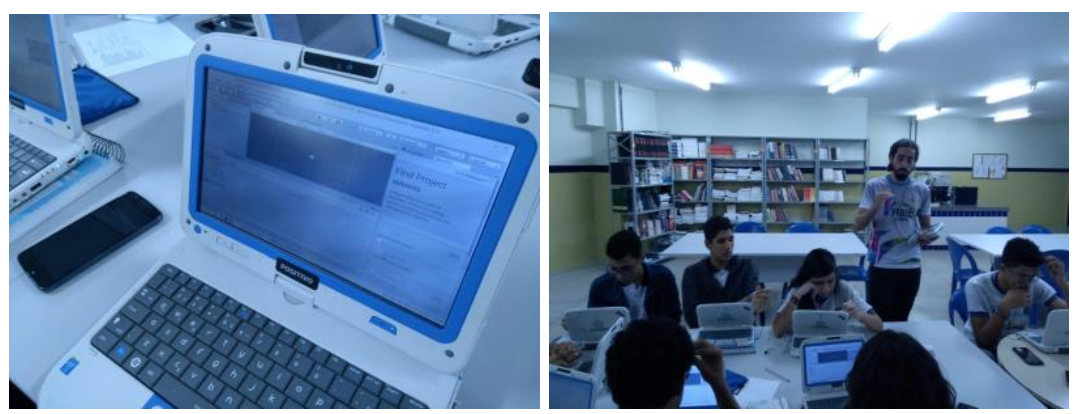

Figura 2. Tablets utilizados em laboratório; Foto 3. Atividade em sala de aula.

\subsection{Integração com as Aulas Curriculares}

Para melhorar a motivação dos alunos e evitar mais desistências, foi proposto pelos professores do currículo técnico aplicar uma atividade somativa nas disciplinas relacionadas à programação, de tal forma que os alunos de fora do clube não fossem excluídos. Os docentes propuseram uma atividade com título Surpreenda-me, pela qual os alunos trouxeram artefatos diferentes do contexto da aula mas que se relacionassem com a programação, assim os participantes do clube puderam mostrar os jogos que desenvolvem no mesmo, ou usar do conhecimento adquirido para produzir outros.

Dentre os alunos que fizeram a atividade, destacou-se um que utilizou os conceitos das aulas no âmbito da lógica do jogo de entradas de usuário, spritess e condições, em um jogo no Scratch ${ }^{\mathrm{TM}}$ que é a ferramenta usada de forma geral pelos alunos do $1^{\circ}$ ano, mas que não é a aplicada no clube. O que demonstra que os conceitos aplicados no clube são mais abstratos do que a simples ferramenta ou linguagem de programação.

\subsection{Artefatos Desenvolvidos ou em Fase de Desenvolvimento}

Para a segunda etapa do projeto ainda em andamento, seguiremos com todos os alunos participantes para o desenvolvimento de jogos a serem idealizados por eles baseado na temática de frevo, visando utilizar as técnicas trabalhadas na primeira etapa do projeto do clube juntamente a uma carga de conteúdos de gerenciamento de projeto, design de jogos, design gráfico e design de som. Eles serão divididos em equipes de 4 a 5 participantes, cada qual estabelecido em uma função necessária para o desenvolvimento dos seus projetos. A ideia é que os alunos, ao final do projeto, tenham a oportunidade de publicar os jogos, promovendo a inicialização da criação de portfólios individuais desde o atual nível escolar.

\section{Considerações Finais}

Pode-se considerar que os principais objetivos do projeto relatado estão parcialmente atingidos. Dado que os alunos relatam maior facilidade nas matérias técnicas como relata um dos alunos em um questionário6 aplicado recentemente. A linguagem de programação C\# não foi a maior dificuldade entre os alunos mesmo sendo a primeiramente vista por alguns deles, ou seja, ela foi uma ferramenta válida em vez de uma causa de dificuldades entre os alunos do grupo, o que é preliminarmente um excelente resultado.

5 Artefato de imagem do jogo.

6 Disponível em:

<https://docs.google.com/spreadsheets/d/1iaPWiNeSo8iZhdIiE6NpYmdZqy4rp6SqvOeQBQ-

$3 \mathrm{dR} 0 /$ edit?usp=sharing $>$ 
Destaca-se que no decorrer do projeto houve desafios, tais como a diminuição expressiva no número de alunos participantes e as limitações dos recursos tecnológicos disponíveis. Apesar disso, os alunos que se mantém participantes são assíduos, dedicados e produtivos. Tal participação também se dá pela colaboração recíproca entre os alunos, dado que muitas vezes tiram as dúvidas uns dos outros.

Quanto ao uso da estratégia de sala de aula invertida, apesar do desafio de manter os alunos interessados e em dia assistindo aos vídeos, foram feitos elogios às videoaulas dentro dos próprios comentários no canal. Além disso, os resultados dos questionários foram importantes para identificar dúvidas antes de iniciar a prática da aula. Deste modo, não se pode considerar que a sala de aula invertida foi a melhor escolha para o cenário de uma escola integral, porém foi de grande importância para diminuir o número de aulas necessárias.

Sendo assim, a expectativa para a segunda etapa do projeto é de que os alunos se organizem em duas ou três equipes em cada turma. Como dito anteriormente, a proposta para a segunda etapa envolve os grupos idealizando os seus próprios projetos e utilizando os conhecimentos adquiridos na primeira etapa sob a orientação dos monitores para desenvolvê-los.

\section{Referências Bibliográficas}

SILVEIRA, S. R., PEREIRA, A. S., BERTOLINI, C., PARREIRA, F., \& BIGOLIN, N. . Educação a distância, sala de aula invertida e aprendizagem baseada em problemas: possibilidades para o ensino de programação de computadores. In ANAIS DOS WORKSHOPS DO CONGRESSO BRASILEIRO DE INFORMÁTICA NA EDUCAÇÃO, 2018, Fortaleza, Anais... Fortaleza: UFC, v. 7, n. 1, p. 1052, out. 2018.

SOUZA, M. V. R., \& FRANÇA, A. C. C.. Um estudo sobre as dificuldades no processo de aprendizagem de programação no Curso de Análise e Desenvolvimento de Sistemas na FAFICA - Faculdade de Filosofia, Ciências e Letras de Caruaru-PE. Revista da Escola Regional de Informática, v. 2, n.2, p.19-27, 2013.

ANDRADE, R., MENDONÇA, J., OLIVEIRA, W., ARAUJO, A. L. \& SOUZA, F. . Uma Proposta de Oficina de Desenvolvimento de Jogos Digitais para Ensino de Programação. ANAIS DOS WORKSHOPS DO V CONGRESSO BRASILEIRO DE INFORMÁTICA NA EDUCAÇÃO (CBIE 2016), [s.1.], p.1127-1136, 2016.

CARVALHO, R J. O. "Virar a Sala de Aula": Centrar a Aprendizagem no Aluno Recorrendo a Ferramentas Cognitivas. Relatório de projeto de mestrado em Ensino de Informática - Universidade do Minho, Braga, p. 369, 2014.

MARQUES, D. L.; COSTA, L. F. S.; SILVA, M. A. A. \& REBOUÇAS, A. D. D. S., Atraindo Alunos do Ensino Médio para a Computação: Uma Experiência Prática de Introdução à Programação utilizando Jogos e Python. Anais Eletrônicos... In: Workshop de Informática na Escola de 2011, Aracaju/SE, 2011. 